\title{
Effect of psychological stress on Tempomandibular joint (Experimental study)
}

\section{Original Article}

Khaled Ibrahim Barakat, Ahmed Abdelmonem Zaki and Mohammad Hussein Zaki

Department of Oral and Maxillofacial Surgery, Faculty of Dentistry, Minia University, Minia, Egypt

\begin{abstract}
Background: Psychological stress is considered a possible pathogenic factor for temporomandibular joint disorders (TMD), but few reports have supplied direct evidence. This study was designed to observe the effects of psychological stress on the TMJ structures.

Materials and Methods: A well-established rat communication box model were used to induce a psychological stress in rats (PS group). The control group was not subjected to psychological insult. After one month, the rats were euthanized and TMJ specimens were harvested to perform a histological analysis under light microscope.

Results: Light microscopy showed apparent pathological changes in the TMJs of psychologically stressed rats.

Conclusion: These results indicate that psychological stress is able to induce pathologic alterations in the TMJ structures and plays an important role in TMD.
\end{abstract}

Key Words: Psychology, Stress, Tempomandibular joint.

Received: 18 February 2020, Accepted: 15 March 2020.

Corresponding Author: Khaled Ibrahim Barakat, Department of Oral and Maxillofacial Surgery, Faculty of Dentistry, Minia University, Minia, Egypt, Tel.: +20222677077, Mobile: +201222324596, E-mail: send2kbarakat@yahoo.com.

ISSN: 2090-097X, July 2019, Vol. 10, No. 3

\section{INTRODUCTION}

The tempromandibular joint (TMJ) is a bilateral synovial articulation that connects the mandible to the skull and permits the lower jaw to move and function. Unlike other joints, the articular portion of the mandibular condyle is composed of fibrocartilage instead of hyaline cartilage $^{[1,2]}$. The fibrocartilaginous tissue of mandibular condyles serves as an important shock-absorbing and lubricating material during physiological activities involving the $\mathrm{TMJ}^{[3,4]}$. The subchondral bone, lies underneath the articular cartilage and is engaged during altered loading. The functional integrity of the two structures allows the TMJ to histomorphologically adapt to mechanical loading ${ }^{[5-7]}$.

Temporomandibular disorder (TMD), a common disease, is a collection of conditions affecting bone and cartilage of $\mathrm{TMJ}^{[8]}$. TMD are characterized by pain, clicking and limited mouth opening which can impair sleep and quality of life ${ }^{[9,10]}$.

There are a wide diversity of contributing factors that participate in aggravating TMD including; psychological problems, occlusal disorders, Para functional habits and autoimmune disorders of the joints ${ }^{[11]}$. Among various contributing factors, the psychological stress involved in the etiology of TMD have remained controversial until Schwartz realized that TMD and psychological factors are closely related ${ }^{[12]}$, then this relation had attracted the attention of many researchers ${ }^{[13-15]}$, to perform clinical studies that showed higher prevalence rates of psychiatric disorders among patients with TMD when compared to healthy people ${ }^{[15]}$. The later researches included psychological conditions as one of the predisposing and perpetuating factors of TMD and this transformed the treatment of TMD from a biomedical to biopsychosocial model ${ }^{[16,17]}$.

Although many scholars have confirmed the epidemiologic relationships between TMD and dysphoria, including depression, anxiety, tension, and stress, none of the studies completely interpreted the mechanism underlying this relationship ${ }^{[18]}$. Proof of the link between psychological factors and TMD was derived mainly from clinical reports, epidemiological studies, or questionnaire surveys, the direct evidence from animal studies is still lacking ${ }^{[19]}$. Therefore, our aim of the study was to provide an animal research to investigate the effect of psychological stress on TMJ.

\section{MATERIALS AND METHODS}

The current study was a prospective randomized controlled experimental study that delivered to female Albino rats from laboratory animal center, faculty of medicine, Minia University to assess the effect of psychological stress on the tempomandibular joint (TMJ). The rats ages range from 2 - 3 months and their weight range from $200-300 \mathrm{gm}$. They were acclimated to laboratory conditions one week before the experiment, with food and water available ad libitum and then randomly divided into 3 groups: 
1. Control group. (CTRL group) (8 rats).

2. Experimental group or Psychological stress group. (PS group) (8 rats).

3. Foot shock group. (FS group) (16 rats).

The control group rats were not subjected to any insult. The experimental group rats were subjected to psychological insult only. The foot shock group were subjected to electric shock to induce psychological insult to experimental group through communication box. The FS rats that received foot shocks merely served as a source of psychological stress for the PS group rats and were not sampled for experimental observation. Eight rats were randomly selected from this group each day to receive a shock in order to minimize anticipation and to prevent adaptation to the stress.

\section{Communication box}

Psychological stress was induced by a communication box (CB) (Figure 1) which was proved to induce a psychological stress in rats and lead to biochemical changes in orofacial muscles and brux like activity in rats ${ }^{[20,21]} \cdot \mathrm{CB}$ is a glass box divided into 16 compartments which separated by transparent glass partitions to allow the rats to see each other without direct contact. Small holes were perforated in the glass partitions to allow proper ventilation. The ground of the box was constructed from a metal grid connected to electric generator to allow delivery of electric shock. Half of the cells ground were alternatively covered by wooden blocks in order to electrically isolate the ground and divide the box into electric conducting and non-electric conducting cells. When the FS group received electric shock, they screamed and jumped which subsequently induced indirect stress insult to the PS group by allowing them to visualize hear and smell the reaction of shocked rats. Electric shock was delivered via a generator with output voltage $48 \mathrm{~V}$ that produced intermittent electric shock every $2 \mathrm{sec}$ for one hour daily for a period of one month.

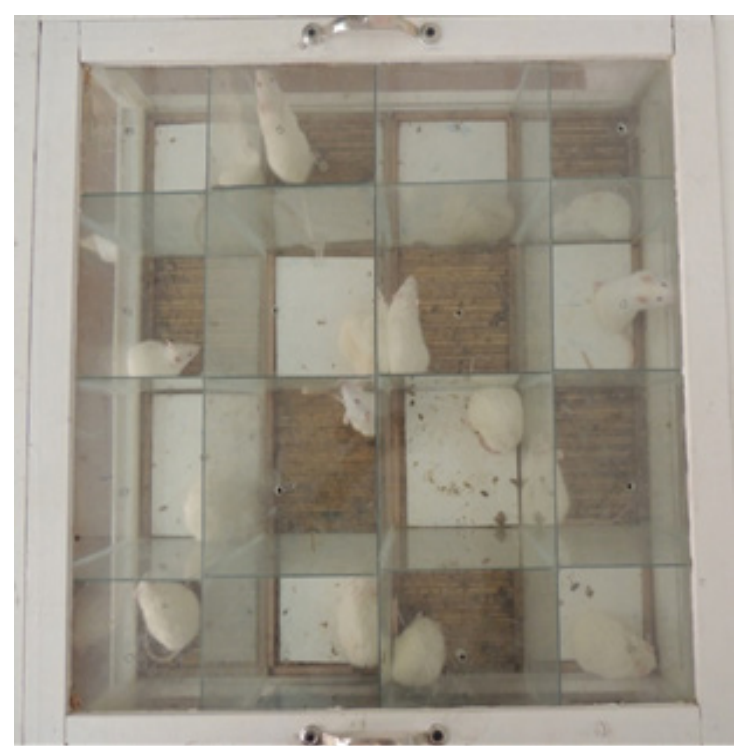

Figure 1: Communication box.
After one-month all rats were euthanized under anesthesia with an intraperitoneal injection of pentobarbital sodium $(50 \mathrm{mg} / \mathrm{kg} \text { body weight })^{[22]}$. The TMJs were stored and preserved in $10 \%$ paraformaldehyde. After preparation, the joint specimens were subjected for histological analysis through light microscope.

\section{RESULTS}

Light microscopy revealed apparent difference between CTRL group and PS group. The disc in the CTRL group appeared with normal and homogenous thickness, while in PS group the disc became apparently thinner with discontinuity of its surface in some specimens. The condyles in the CTRL group showed homogenous distribution of its layers, smooth articular surface, and chondrocytes normally distributed throughout the cartilage, while in PS group condyles showed collapsing of its layers and infiltration of many inflammatory cells observed (Figure 2).

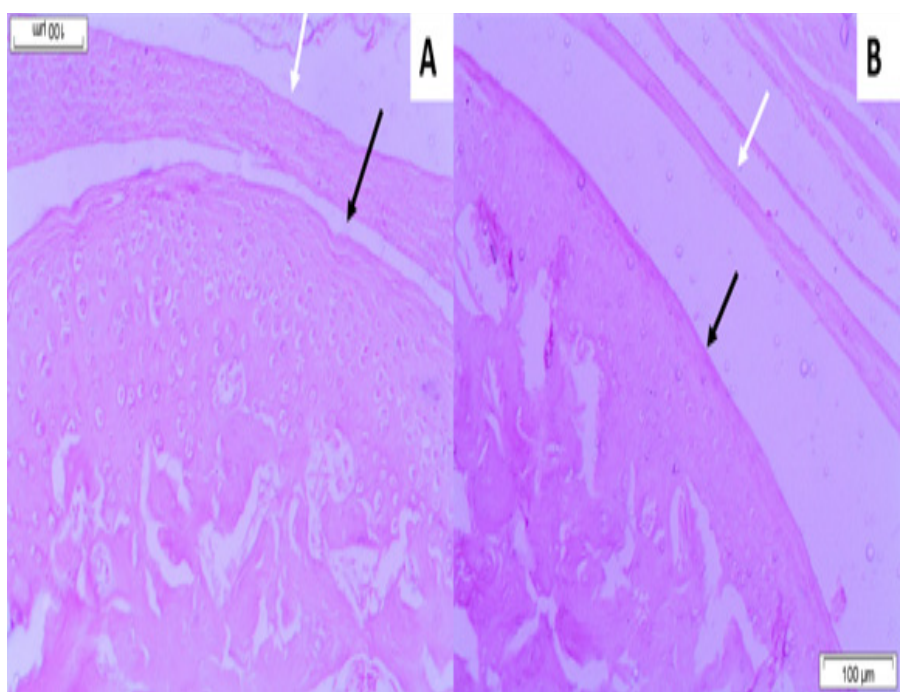

Figure 2: Sagittal sections of the rats TMJ of CTR1 group (A) and PS group (B) stained with $\mathrm{H} \& \mathrm{E}$ as viewed under light microscope. white arrow points to the articular disc, black arrow points to the condyle.

\section{DISCUSSION}

TMD is a common stomatological disorder that affects $20-40 \%$ of the population ${ }^{[23]}$. Numerous studies have illustrated the multifactorial causes of TMD. Among various factors, psychological stress had increased interest in several researches that were mainly clinical studies. Thus, our aim was to perform an experimental study on rats to provide a higher research evidence to explore the relation between psychological stress and TMD. The experimental research provided several advantages, as it allowed us to investigate the effect of a single factor on 
TMJ while excluding the effects of other factors and this precise selective investigation was beneficial in studying a disease like TMD with a multifactorial etiology. In addition, the animal research permitted performing a histological analysis from extracted specimens which reflect the actual pathological changes on the cellular level.

TMD in humans is more common in females, suggesting the sex-related factors in the etiology, only female rats were used in this study to avoid gender differences ${ }^{[24,25]}$.

We used the communication box which is a wellestablished method for introducing psychological stress to animals ${ }^{[20,21]}$. Communication box generated an electric current causing intermittent electric shock to FS group rendering them to jump and scream which induced a pure psychological stress into PS group without direct physical contact. The rats were exposed to psychological stress for one hour daily for one month which was considered a sufficient time to cause anxious effect on rats according to previous studies ${ }^{[26,27]}$.

We noticed behavioral changes of PS group throughout the experiment. The PS rats were trying to keep apart from adjacent PS rats, they avoid visual contact with them and turning their faces to the opposite corner. The food intake of PS rats decreased and their body weight diminished during the experiment. These changes reflected the anxious state created by the communication box to PS rats.

We used light microscope to investigate the histological changes of the disc and condylar surface to prove that psychological stress can induce pathological changes to the TMJ structures in the cellular level. The histological analysis revealed apparent changes of the disc and condylar surface. the disc became thinner and discontinuity of its surface appeared in some slides. Debonding of the fibrous layer (the most superficial layer of the condyle) was noticed in many samples. There was also numerous inflammatory cell infiltration in the connective tissue of the condyle.

The current study provided only one method of analysis which was light microscopic examination. Further methods are required such as electron microscopy and measuring inflammatory cytokines to provide a thorough analysis of psychological stress effect on TMJ.

According to previous results we conclude that psychological stress caused structural changes to articular surfaces of TMJ in rats and we can consider PS as initiative and progressive factor for TMD that should be reflected in diagnosis and treatment of TMD.

\section{CONFLICT OF INTEREST}

The authors declare no conflict of interest.

\section{REFERENCES}

1. Benjamin, M. and J.R. Ralphs, Biology of fibrocartilage cells. Int Rev Cytol, 2004. 233: p. $1-45$.

2. Barakat, K., Correlation between pain and arthroscopic features of synovitis: A logical approach to verify correlations of TMJ pain. Tanta Dental Journal, 2013. 10(3): p. 168 - 172.

3. Hu, K., et al., Effects of condylar fibrocartilage on the biomechanical loading of the human temporomandibular joint in a three-dimensional, nonlinear finite element model. Med Eng Phys, 2003. 25(2): p. 107 - 13.

4. Tanaka, E., et al., Dynamic compressive properties of the mandibular condylar cartilage. J Dent Res, 2006. 85(6): p. $571-5$.

5. Papachristou, D.J., et al., Functional alterations in mechanical loading of condylar cartilage induces changes in the bony subcondylar region. Arch Oral Biol, 2009. 54(11): p. 1035 - 45.

6. Sriram, D., et al., Effects of mechanical stimuli on adaptive remodeling of condylar cartilage. $\mathrm{J}$ Dent Res, 2009. 88(5): p. 466 - 70.

7. Amin, M.F., A.M. Hassan, and K.I. Barakat, The accuracy of dynamic Magnetic Resonance Imaging in evaluation of internal derangement of the temporomandibular joint; comparison with arthroscopic findings. The Egyptian Journal of Radiology and Nuclear Medicine, 2012. 43(3): p. 429 - 436.

8. Carlsson, G.E., Epidemiology and treatment need for temporomandibular disorders. J Orofac Pain, 1999. 13(4): p. $232-7$.

9. Natu, V.P., et al., Temporomandibular disorder symptoms and their association with quality of life, emotional states and sleep quality in SouthEast Asian youths. J Oral Rehabil, 2018. 45(10): p. $756-763$.

10. Exposto, F.G., T. Arima, and P. Svensson, Sleep Disorders and Chronic Orofacial Pain. Current Sleep Medicine Reports, 2019. 5(3): p. 104 - 111.

11. Slade, G.D., et al., Influence of psychological factors on risk of temporomandibular disorders. J Dent Res, 2007. 86(11): p. 1120 - 5. 
12. Weinberg, L.A., The role of stress, occlusion, and condyle position in TMJ dysfunction-pain. J Prosthet Dent, 1983. 49(4): p. 532 - 45.

13. Macfarlane, T.V., J. Kincey, and H.V. Worthington, The association between psychological factors and oro-facial pain: a community-based study. Eur J Pain, 2002. 6(6): p. 427 - 34.

14. Kanehira, H., et al., Association between stress and temporomandibular disorder. Nihon IIoteri Shika Gakkai Zasshi 2008: 52: 37580-. Rossetti LM, Rossetti PH, Conti PC, deAraujo Cos R.Association between sleep bruxism and temporomandibular disorders: a polysomnographic pilot study. Cranio, 2008. 26: p. $16-24$.

15. Gatchel, R.J., et al., Major psychological disorders in acute and chronic TMD: an initial examination. $\mathrm{J}$ Am Dent Assoc, 1996. 127(9): p. $13651374,1372,70-$.

16. McNeill, C., Management of temporomandibular disorders: concepts and controversies. The Journal of prosthetic dentistry, 1997. 77(5): p. 510 - 522.

17. LeResche, L., et al., Predictors of onset of facial pain and temporomandibular disorders in early adolescence. Pain, 2007. 129(3): p. 269 - 78.

18. Manfredini, D., et al., A critical review on the importance of psychological factors in temporomandibular disorders. Minerva Stomatol, 2003. 52(6): p. 32130-327,6-.

19. Wu, G., et al., Psychological stress may contribute to temporomandibular joint disorder in rats. J Surg Res, 2013. 183(1): p. 223 - 9.

20. Li, Q., et al., Oxidative damage and HSP70 expression in masseter muscle induced by psychological stress in rats. Physiol Behav, 2011. 104(3): p. $365-72$.

21. Rosales, V.P., et al., Emotional stress and bruxlike activity of the masseter muscle in rats. Eur $\mathrm{J}$ Orthod, 2002. 24(1): p. 107 - 17.

22. Leary, S.L., et al. AVMA guidelines for the euthanasia of animals: 2013 edition. 2013. American Veterinary Medical Association Schaumburg, IL.

23. Christidis, N., et al., Prevalence and treatment strategies regarding temporomandibular disorders in children and adolescents-A systematic review. Journal of oral rehabilitation, 2019. 46(3): p. 291301-.

24. Berger, M., et al., Association between estrogen levels and temporomandibular disorders: a systematic literature review. Przeglad menopauzalny $=$ Menopause review, 2015. 14(4): p. 260.

25. Lövgren, A., et al., A high prevalence of TMD is related to somatic awareness and pain intensity among healthy dental students. Acta Odontologica Scandinavica, 2018. 76(6): p. 387 - 393.

26. Wu, G., et al., Psychological stress induces alterations in temporomandibular joint ultrastructure in a rat model of temporomandibular disorder. Oral Surg Oral Med Oral Pathol Oral Radiol Endod, 2011. 112(6): p. e106 - 12.

27. Li, Q., et al., Psychological stress alters microstructure of the mandibular condyle in rats. Physiol Behav, 2013. 110111-: p. 129 - 39. 